\title{
A PARTITION RELATION FOR SOUSLIN TREES ${ }^{1}$
}

\author{
BY
}

\author{
ATTILA MÁTÉ
}

\begin{abstract}
The aim of these notes is to give a direct proof of the partition relation Souslin tree $\rightarrow(\alpha)_{k}^{2}$, valid for any integer $k$ and any ordinal $\alpha<\omega_{1}$. This relation was established by J. E. Baumgartner, who noticed that it follows by a simple forcing and absoluteness argument from the relation $\omega_{1} \rightarrow(\alpha)_{k}^{2}$, which is a special case of a theorem of Baumgartner and A. Hajnal.
\end{abstract}

1. Introduction. Let $\langle P,\langle\rangle$ be a partial order, and let $r>0$ be an integer. Then $[\langle P,<\rangle\}^{r}$, usually abbreviated as $[P]^{r}$, denotes the set of all sequences $\left\langle x_{1}, \ldots, x_{r}\right\rangle$ of elements $x_{1}<\cdots<x_{r}$ of $P$; note that this is an unusual definition of $[P]^{r}$. If $\alpha$ is an ordinal and $k, r>0$ are integers, then the symbol $P \rightarrow(\alpha)_{k}^{r}$ expresses that given any partition $K=\left\langle K_{1}, \ldots, K_{k}\right\rangle$ of $[P]^{r}$ into $k$ parts, i.e.

$$
[P]^{r}=K_{1} \cup \cdots \cup K_{k}
$$

there is an $i$ with $1 \leqslant i \leqslant k$ and a set $X \subset P$ of order type $\alpha$ such that $[X]^{r} \subset K_{i}$ (such an $X$ is called homogeneous for $K$ ). If $P$ is a total order of order type $\varphi$, then usually $\varphi \rightarrow(\alpha)_{k}^{2}$ is written instead of $P \rightarrow(\alpha)_{k}^{2}$. The negation of the above relation is expressed by substituting $\nrightarrow$ for $\rightarrow$. Order type will always refer to total orders, and tp $X$ will denote the order type of the totally ordered set $X$.

Baumgartner and Hajnal [1] proved that if $\varphi$ is an order type such that $\varphi \rightarrow(\omega)_{\omega}^{1}$, then $\varphi \rightarrow(\alpha)_{k}^{2}$ holds for any ordinal $\alpha<\omega_{1}$ and any integer $k>0$. Their proof proceeded by first establishing the result with the aid of Martin's Axiom, and then by showing via absoluteness considerations that the result held in any well-founded model of set theory. F. Galvin [3] gave a direct combinatorial proof of this result; his proof, in fact, extended this result so as to apply to certain partially ordered sets. His proof, however, did not cover the case of Souslin trees (the definition will be given below), while Baumgartner observed that the relation Souslin tree $\rightarrow(\alpha)_{k}^{2}$ $\left(\alpha<\omega_{1}, 0<k<\omega\right)$ follows by a simple forcing and absoluteness argument from the relation $\omega_{1} \rightarrow(\alpha)_{k}^{2}$, which is a special case of the Baumgartner-Hajnal theorem mentioned above; c.f. [3, Theorem 11, p. 11], although really no details are given there. For the benefit of the reader, we give some details: What one does is one

Received by the editors September 21, 1980.

1980 Mathematics Subject Classification. Primary 03E05, 04A20; Secondary 03E40.

Key words and phrases. Infinite game, partition relation, Souslin tree.

'This paper was written during the author's stay at the Institute for Advanced Study, Princeton, New Jersey, in the summer of 1980, and presented at the meeting of the Association for Symbolic Logic in San Francisco, California, in January 1981. It is based upon work supported by the National Science Foundation under Grant No. MCS 7901732. 0002-9947/81/0000-0506/\$02.75 
uses the Souslin tree as a notion of forcing, which in the extended model will have a chain of length $\omega_{1}$. Then one obtains a homogeneous set of type $\alpha$ by the relation $\omega_{1} \rightarrow(\alpha)_{k}^{2}$ in the extended model. Using an absoluteness argument due to J. $\mathbf{H}$. Silver (cf. [1, §2, p. 199]), one can then establish that the ground model also has a homogeneous set of type $\alpha$.

In these notes we are going to give a direct proof of the result involving Souslin trees. The desire for finding such a proof was expressed by Galvin, and he speculated that such a proof might shed some light on the conjectures on $\mathrm{pp}$. 719-720 of his paper [3]. While this may be the case, at this point we are unable to decide any of those conjectures. Still, the points where the proof breaks down when trying to apply it to establish, say, his Conjecture $3^{\prime}$ can be readily identified, and this may be helpful to future investigators. The same thing does not seem to apply to the metamathematical proof of Baumgartner outlined above.

The proof given below relies heavily on Galvin's proof, but it is somewhat more complicated. The results of $\S \S 3$ and 4 of his paper are used without any change; they are restated for the convenience of the reader, but their proofs are not given.

2. Souslin trees. A chain is a totally ordered subset of a partial order. A partial order is well-founded if it has no infinite descending chain. A well-founded partial order is a tree if, for any element, the set of its predecessors is totally ordered (and so well-ordered as well). An antichain in a tree is a set of pairwise incomparable elements. An uncountable tree is called a Souslin tree if it has no uncountable chain or antichain. The existence of a Souslin tree is undecidable from the usual axioms of set theory; see e.g. the book [4] for details. (The spelling there is Suslin.)

A Souslin tree $\langle P,\langle\rangle$ will be called flourishing if the set $\{y \in P: x<y\}$ of successors of $x$ is uncountable for any $x \in P$. It is fairly easy to see that if $P$ is any Souslin tree then the subtree

$$
\{x \in P:\{y \in P: x<y\} \text { is uncountable }\}
$$

is a flourishing Souslin tree.

Let $\langle P,\langle\rangle$ be a Souslin tree, $p \in P$. A set $X \subset P$ is said to be dense above $p$ if for any $q>p$ there is an $x \in X$ with $q<x$. The following is well known.

Lemma 2.1. Let $\langle P,\langle\rangle$ be a Souslin tree, and let $X \subset P$ be uncountable. Then there is a $p \in P$ such that $X$ is dense above $p$.

Proof. By Zorn's lemma, there is a set $Q$ such that (a) $Q$ is an antichain, (b) for any $q \in Q$, there is no $x \in X$ with $q<x$, and (c) if $p$ is any element of $P$, then $p$ is either (i) comparable to some element of $Q$, or (ii) there is an $x \in X$ with $p<x$. Now if $Q$ is a maximal antichain, it is easy to see from (b) that $X$ is countable, which contradicts our assumptions. If not, then there is $p \in P$ that is not comparable to any element of $Q$. Then no $p^{\prime}>p$ is comparable to any element of $Q$ either, hence $p^{\prime}<x$ for some $x \in X$ according to (c). That is, $X$ is dense above $p$, which is what we wanted to prove. 
Our aim is to give a direct combinatorial proof of

THeOREM 2.2 (J. E. BAUMgartner). If $P$ is a Souslin tree then

$$
P \rightarrow(\alpha)_{k}^{2}
$$

holds for any countable ordinal $\alpha$ and any positive integer $k$.

To this end we may assume that $P$ is a flourishing Souslin tree; fix $P$ and a partition $K$ with $K_{1} \cup \cdots \cup K_{k}=[P]^{2}$. We have to show that there are homogeneous sets of any countable order type for this partition.

3. Almost homogeneous chains. We will define a weak homogeneity property which will enable us to construct large homogeneous sets. The results are given without proofs here, and their proofs can be found in Galvin's paper [3, §3, pp. 720-722].

For $A, B \subset P$, write $A<B$ if $a<b$ for any $a \in A$ and $b \in B$. $a<B$ and $A<b$ will have analogous meanings. A chain $X \subset P$ is called almost homogeneous (for $K$ ) in case the following condition is satisfied: given any ordinals $\beta<\alpha<\omega_{1}$ and any set $A \subset X$ with tp $A=\omega^{\alpha}$, there exist $B, A^{\prime} \subset A$ and $i, 1 \leqslant i \leqslant k$, such that $B<A^{\prime}, \operatorname{tp} B=\omega^{\beta}, \operatorname{tp} A^{\prime}=\omega^{\alpha}$, and $B \times A^{\prime} \subset K_{i}$. Let $\mathcal{H}$ denote the set of all almost homogeneous chains of $P$.

Lemma 3.1. (i) If $X \in \mathcal{H}$ and $Y \subset X$, then $Y \in \mathcal{H}$.

(ii) Suppose $X=\cup_{n<\omega} X_{n}$, where $X_{n} \in \mathcal{H}$, and for each $m<\omega$ there is an $i$ with $1 \leqslant i \leqslant k$ such that $X_{m} \times X_{n} \subset K_{i}$ for all $n>m$. Then $X \in \mathcal{H}$.

(iii) For every $\alpha<\omega_{1}$ there exists a $\beta<\omega_{1}$ such that, for any $B \in \mathcal{H}$ with $\operatorname{tp} B=\omega^{\beta}$ there is $A \subset B$ with $\operatorname{tp} A=\omega^{\alpha}$ and $[A]^{2} \subset K_{i}$ for some $i, 1 \leqslant i \leqslant k$.

For the proof, see [3, pp. 720-722]; (iii) gives the rationale for considering almost homogeneous chains.

4. The notion $G(\alpha, i)$. Given $A \subset P$ and $x \in P$ with $\operatorname{tp} A=\omega^{\alpha}$ and $A<x$, it is relatively easy to see that there are $A^{\prime} \subset A$ with $\operatorname{tp} A^{\prime}=\omega^{\alpha}$ and $i$ with $1 \leqslant i<k$ such that $A^{\prime} \times\{x\} \subset K_{i}$. This observation is, however, of limited usefulness unless one is able to anticipate the value of $i$. This is the purpose of the concept $G(\alpha, i)$, defined for $\alpha<\omega_{1}$ and $1 \leqslant i \leqslant k$. The definition is given by recursion on $\alpha$.

$G(0, i)$ is the set of all pairs $\langle\{a\}, X\rangle$ such that $a \in P, X \subset P$ is uncountable and $\{a\} \times X \subset K_{i}$. Now let $0<\alpha<\omega_{1}$ and suppose that $G(\beta, i)$ has been defined for all $\beta<\alpha$. Then $G(\alpha, i)$ is the set of all pairs $\langle A, X\rangle$ such that $A \subset P$ is a chain, tp $A=\omega^{\alpha}, X \subset P$ is uncountable, and the following condition is satisfied: for any $A^{\prime} \subset A$ with tp $A^{\prime}=\omega^{\alpha}$, any uncountable $X^{\prime} \subset X$, and any $\beta<\alpha$, there exist $B \subset A^{\prime}$ and uncountable $X^{\prime \prime} \subset X^{\prime}$ such that $B$ is bounded in $A^{\prime}$ and $\left\langle B, X^{\prime \prime}\right\rangle \in G(\beta, i)$.

The proof of the following lemma is given in $[3, \S 4$, pp. $722-723] ; \Sigma$ in that paper is to be taken as the set of all uncountable subsets of $\boldsymbol{P}$ for our purpose. 
Lemma 4.1. (i) If $\langle A, X\rangle \in G(\alpha, i), A^{\prime} \subset A$, tp $A^{\prime}=\omega^{\alpha}$, and $X^{\prime} \subset X$ is uncountable, then $\left\langle A^{\prime}, X^{\prime}\right\rangle \in G(\alpha, i)$.

(ii) Let $\alpha<\omega_{1}$. Suppose that $A \subset P$ is a chain of type $\omega^{\alpha}, X \subset P$ is uncountable, and $A<X$. Then there exist $A^{\prime} \subset A, X^{\prime} \subset X$, and $i$ with $1 \leqslant i \leqslant k$ such that $\left\langle A^{\prime}, X^{\prime}\right\rangle \in G(\alpha, i)$.

(iii) Suppose $\left\langle A_{n}, X\right\rangle \in G\left(\alpha_{n}, i_{n}\right)$ for each $n<\omega$. Then there exist $x \in X$ and $A_{n}^{\prime} \subset A_{n}(n<\omega)$ such that tp $A_{n}=\omega^{\alpha_{n}}$ and $A_{n}^{\prime} \times\{x\} \subset K_{i_{n}}$ for each $n<\omega$.

5. Nets. Galvin introduced a variant of a scheme due to P. Erdös, A. Hajnal, and E. C. Milner [2, \$3, pp. 332-335] ensuring that an infinite sequence of sets of type $\omega^{\alpha}$ will have intersection of type $\omega^{\alpha}$. We will describe Galvin's scheme, but will refer to his paper for proofs.

For each nonzero ordinal $\alpha<\omega_{1}$ choose a finite sequence $\langle\alpha(n): n<\omega\rangle$ of ordinals such that $\omega^{\alpha}=\sum_{n<\omega} \omega^{\alpha(n)}$ and $\alpha(n) \leqslant \alpha(n+1)$ for $n<\omega$.

A net is a finite set $\Re$ of subsets of $P$ such that (1) $\cup \mathfrak{N}$ is a chain; (2) for each $A \in \mathfrak{N}$ there is an $\alpha<\omega_{1}$ such that $\operatorname{tp} A=\omega^{\alpha}$; (3) if $A, B \in \mathfrak{N}$ and $A \neq B$, then either $A<B$ or $B<A$.

Let $\mathfrak{R}$ and $\mathfrak{R}^{\prime}$ be nets. $\mathfrak{R}^{\prime}$ is a refinement of $\mathfrak{R}$; in symbols $\mathfrak{N}^{\prime} \leqslant \mathfrak{N}$ if (1) for each $A^{\prime} \in \mathfrak{N}^{\prime}$, there is an $A \in \mathfrak{R}$ such that $A^{\prime} \subset A$; (2) for each $A \in \mathfrak{R}$, there is an $A^{\prime} \in \mathfrak{N}^{\prime}$ such that $A^{\prime} \subset A$ and tp $A^{\prime}=\operatorname{tp} A$.

Let $\mathfrak{N}, \mathfrak{N}^{\prime}$ be nets, and let $n<\omega . \mathfrak{N}^{\prime}$ is an $n$-refinement of $\mathfrak{N}$, in symbols $\mathfrak{N}^{\prime}<^{n} \mathfrak{N}$, if $\mathfrak{N}^{\prime} \leqslant \mathfrak{N}$ and the following additional condition is satisfied: if $A \in \mathfrak{N}, \operatorname{tp} A=\omega^{\alpha}$, $\alpha>0$, then there is a $B \in \mathfrak{N}^{\prime}$ such that $B \subset A$ and $\omega^{\alpha(n)} \leqslant \operatorname{tp} B<\omega^{\alpha}$.

A refining sequence is a sequence $\left\langle\mathfrak{N}_{i}: i\langle\omega\rangle\right.$ of nets such that: (1) $\mathfrak{N}_{i+1} \leqslant \mathfrak{N}_{i}$ for all $i<\omega$; (2) for any $i<\omega$ and any $n<\omega$, there is a $j<\omega$ such that $\mathfrak{N}_{j}<^{n} \mathfrak{N}_{i}$.

Lemma 5.1. Let $\left\langle\mathfrak{R}_{i}: i\langle\omega\rangle\right.$ be a refining sequence. $S_{i}=\cup \mathfrak{N}_{i}, S=\bigcap_{i<\omega} S_{i}$. Then, for any $A \in \cup_{i<\omega} \mathfrak{N}_{i}$ we have $\operatorname{tp}(A \cap S)=\operatorname{tp} A$. (In particular, if $\mathfrak{N}_{0}=\{A\}$, then $\operatorname{tp} S=\operatorname{tp} A$.)

The proof is relatively simple, proceeds by induction on the order type of $A$, and can be found in [3, Lemma 16, p. 726].

6. Constructing a large almost homogeneous set. For $i$ with $1 \leqslant i \leqslant k$ let $G(i)$ be the set of all pairs $\langle\mathfrak{N}, X\rangle$ where $X \subset P$ is uncountable and $\mathfrak{R}$ is a net such that, for each $A \in \mathfrak{N}$, we have $\langle A, X\rangle \in G(\alpha, i)$ for some $\alpha<\omega_{1}$. The following lemma is obvious.

Lemma 6.1. If $\langle\mathfrak{R}, X\rangle \in G(i)$ then, for any $n\left\langle\omega\right.$, there is $\left\langle\mathfrak{N}^{\prime}, X^{\prime}\right\rangle \in G(i)$ such that $X^{\prime} \subset X$ and $\mathfrak{N}^{\prime}<^{n} \mathfrak{R}$.

Our main result, Theorem 2.2, will follow from the following

Lemma 6.2. Let $\beta<\omega_{1}$. Suppose $X \subset P$ is uncountable and $\left\langle A_{r}, X\right\rangle \in G\left(\alpha_{r}, i_{r}\right)$ for $r<N$. Furthermore, suppose that

$$
\bigcup_{r<N} A_{r}<X
$$

Then there exist $B \cup\{b\} \subset X$ and $A_{r}^{\prime} \subset A_{r}(r<N)$ such that $B \in \mathcal{H}, \operatorname{tp} B=\omega^{\beta}$, $B<b, \operatorname{tp} A_{r}^{\prime}=\omega^{\alpha_{r}}$, and $A_{r}^{\prime} \times B \subset K_{i_{r}}$ for $r<N$. 
Assumption (2) is convenient but not necessary. First we show how to prove Theorem 2.2 from this result. The rest of the section will be devoted to proving Lemma 6.2.

Proof of Theorem 2.2. Consider any $\alpha<\omega_{1}$. By Lemma 3.1(iii), there is a $\beta<\omega_{1}$ such that an almost homogeneous chain of type $\omega^{\beta}$ ensures the existence of a homogeneous chain of type $\omega^{\alpha}$. Lemma 6.2 with $N=0$ ensures the existence of the former. Thus, the existence of a homogeneous chain of type $\omega^{\alpha}$ follows, which is what we wanted to prove.

Proof of Lemma 6.2. We use induction on $\beta$; i.e. we assume that the assertion of the lemma is true for $\beta^{\prime}<\beta$ and for any $N$. The case $\beta=0$ is contained in Lemma 4.1(iii), so we can assume $0<\beta<\omega_{1}$. Note the following consequence of the induction hypothesis: if $\gamma<\beta, X \subset P$ is uncountable, $\left\langle\mathfrak{N}_{r}, X\right\rangle \in G\left(i_{r}\right)(r<l)$, and

$$
\bigcup_{r<l}\left(\cup \mathfrak{R}_{r}\right)<X
$$

then there exist $B \cup\{b\} \subset X$ and subnets $\mathfrak{M}_{r} \leqslant \mathfrak{N}_{r}(r<l)$ such that $B \in \mathcal{H}$, tp $B=\omega^{\gamma}, B<b,\left\langle\mathfrak{M}_{r}, X\right\rangle \in G\left(i_{r}\right)$ (cf. Lemma 4.1(i)), and $\left(\cup \mathfrak{M}_{r}\right) \times B \subset K_{i_{r}}$ for $r<l$.

Now let $0<\beta<\omega_{1}$ and assume the induction hypothesis. Consider the following game, played by two players, White and Black. The game consists of an $\omega$-sequence of alternate moves by the players; the concept of winning will be defined later. The $l$ th position $(l \geqslant N)$ with White on move consists of nets $\mathfrak{R}_{r}^{l}$ $(r<l)$, an element $p^{l} \in P$ with

$$
\bigcup_{r<l} \cup \mathfrak{N}_{r}^{l}<p^{l}
$$

a set $X^{l}$ with $p^{l}<X^{l}$ such that $X^{l}$ is dense above $p^{l}$, and integers $i_{r}$ such that $\left\langle\mathfrak{N}_{r}^{l}, X^{l}\right\rangle \in G\left(i_{r}\right)$.

White's move is simply to pick a $q^{l+1}>p^{l}$. Then Black, using the induction hypothesis, picks a set $B \cup\{b\} \subset X^{l}$ with $q^{l+1}<B<b, B \in \mathcal{H}$, and tp $B=$ $\omega^{\beta(l)}$ (cf. §5) and nets $\mathfrak{M}_{r}$ such that $\mathfrak{M}_{r} \leqslant \mathfrak{N}_{r}^{l},\left\langle\mathfrak{M}_{r}, X^{l}\right\rangle \in G\left(i_{r}\right)$, and $\left(\cup \mathfrak{M}_{r}\right) \times B$ $\subset K_{i_{r}}(r<l)$. Using Lemma 4.1(ii), then Black picks an $i_{l}$, a $B^{\prime} \subset B$ and a $Y \subset X^{l}$ such that $b<Y$ and $\left\langle B^{\prime}, Y\right\rangle \in G\left(\beta(l), i_{l}\right)$. Write $\mathfrak{M}_{l}=\left\{B^{\prime}\right\}$. Next, using Lemma 6.1 (and Lemma 4.1(i)), Black picks nets $\mathfrak{N}_{r}^{l+1}<^{l} \mathfrak{M}_{r}(r \leqslant l)$ and a set $Y^{\prime} \subset Y$ such that $\left\langle\mathfrak{N}_{r}^{l+1}, Y^{\prime}\right\rangle \in G\left(i_{r}\right)$. Next, according to Lemma 2.1 , he picks a $p^{l+1}>b$ such that $Y^{\prime}$ is dense above $p^{l+1}$ and writes

$$
X^{l+1}=\left\{p \in Y^{\prime}: p>p^{l+1}\right\}
$$

As $P$ was assumed to be flourishing (cf. $\S 2$ ), $X^{l+1}$ is uncountable, and so $\left\langle\mathfrak{N}_{r}^{l+1}, X^{l+1}\right\rangle \in G\left(i_{r}\right)(r \leqslant l)$ in virtue of Lemma 4.1(i). Now Black produced the $(l+1)$ st position, and it is White's move. Note that

$$
q^{l+1}<\cup \mathfrak{R}_{l}^{l+1}(<b)<p^{l+1} .
$$

The game starts with $l=N$ and $\mathfrak{N}_{r}^{N}=\left\{A_{r}\right\}$. By Lemma 2.1, pick an arbitrary $p^{N} \in X$ such that $X$ is dense above $p^{N}$, and write

$$
X^{N}=\left\{p \in X: p>p^{N}\right\}
$$


Then $X^{N}$ is uncountable as $P$ was assumed to be flourishing, and the game can start with the initial position $\left\langle\mathfrak{R}_{r}^{N}, X^{N}\right\rangle(r<N)$ and $p^{N}$. For the sake of simplicity, we may assume that

$$
P=\left(\bigcup_{r<N} A_{r}\right) \cup\left\{p^{N}\right\} \cup X^{N}
$$

When this game has been played through an $\omega$-sequence of moves, we obtain the integers $i_{r}$ and the nets $\Re_{r}^{l}(r<\omega, N \leqslant l<\omega$ and $r<l)$ such that the set

$$
B_{r}=\bigcap_{l>r} \cup \mathfrak{N}_{r}^{l}
$$

has order type $\alpha_{r}$ for $r<N$ and $\beta(r)$ for $r \geqslant N$ according to Lemma 5.1, and such that $B_{r} \times B_{n} \subset K_{i_{r}}$ for $r<n<\omega$. Write $A_{r}^{\prime}=B_{r}$ for $r<N$, and put

$$
B=\bigcup_{N<r<\omega} B_{r} \text {. }
$$

Then $B$ is almost homogeneous by Lemma 3.1(ii), tp $B=\omega^{\beta}$, and all the requirements of the lemma to be proved are satisfied except that we have to find a $b \in P$ such that $B<b$ (cf. (4)). Call the game a win for White if such a $b$ exists; otherwise, call it a win for Black. Now, in order to establish the lemma, all we have to prove is that Black does not have a winning strategy in this game. Here, a strategy for Black is a function on the set of all possible positions with Black on move whose value is Black's move in that position. The statement that Black has no winning strategy means that once such a function is specified, White (knowing this function) can pick his moves in such a way that he wins the game (this of course, means much less than saying that White has a winning strategy, which we do not claim, though it would be interesting to know whether or not this is the case). Such a win for White produces the $b \in P$ with $B<b$, as required.

To show that Black has no winning strategy, assume that he plays the game according to a fixed strategy. White tries different sequences of moves in such a way that, in the different courses of the game, Black, on the lth move, produces a maximal antichain $P^{l}$ of $p^{l}$ 's $(l>N)$ such that different sequences $\left\langle q^{N}, \ldots, q^{l}\right\rangle$ of White's moves lead to different $p$ 's. Assume this is already true for $l=m$; we are then going to show how this can be achieved for $l=m+1(>N)$. Note that for $l=N, P^{N}=\left\{p^{N}\right\}$ forms a maximal antichain in view of (4) ( $p^{N}$, however, was not produced by a move of Black). Assume that, in the different sequences of the game, Black has already played $p_{\eta}^{m+1}, \eta<\gamma$, on the $(m+1)$ st move in such a way that $P_{\gamma}^{m+1}=\left\{p_{\eta}^{m+1}: \eta<\gamma\right\}$ is an antichain. If $P_{\gamma}^{m+1}$ is a maximal antichain, then there is nothing more to do. If not, choose a $q_{\gamma}$ as White's $(m+1)$ st move in such a way that (i) $q_{\gamma}$ exceeds some element of $P^{m}$ and (ii) it is incomparable to all elements $P_{\gamma}^{m+1}$. It is possible to satisfy these conditions since $P^{m}$ was assumed to be a maximal antichain. In view of (i), $q_{\gamma}$ is a continuation of a sequence of moves previously played. Let $p_{\gamma}^{m+1}$ be White's response to $q_{\gamma}$ according to his strategy. As $p_{\gamma}^{m+1}>q_{\gamma}$ (cf. (3)), $\left\{p_{\eta}^{m+1}: \eta<\gamma+1\right\}$ is an antichain. For some $\lambda<\omega_{1}, P^{m+1}=$ $\left\{p_{\eta}^{m+1}: \eta<\lambda\right\}$ is a maximal antichain, which is what we wanted to establish. 
Now let $b \in P$ be such that it exceeds one element of each of the maximal antichains $P^{m}, m<\omega$. There is such a $b$; in fact, all but countably many elements of $P$ are like that. Let

$$
\left\{p^{l}\right\}=P^{l} \cap\{x \in P: x<b\} \quad(l>N) .
$$

From $p^{l}$, determine the move $q^{l}$ of White to which Black responded with $p^{l}$. This is possible, since, as is clear from the construction, there was exactly one move $q^{l}$ of White to which the response $p^{l}$ was made. Now using the sequence $\left\langle q^{l}: N<l<\right.$ $\omega\rangle$ of White's moves and Black's strategy, the full moves $\left\langle\mathfrak{N}_{r}^{l}, X^{l}\right\rangle(r<l), i_{l-1}$, and $p^{l}$ made by Black can be determined. The set $B$ obtained in this course of the game is such that $B<b$, a win for White, showing that the chosen strategy of Black was not a winning strategy. This proves Lemma 6.2.

Note that in the above argument it would not have sufficed to ensure that White's $l$ th moves $q^{l}$ form a maximal antichain, since that does not ensure that the $p^{l}$ 's form a maximal antichain. Moreover, the $q$ 's above do not necessarily form an antichain. All that is true is that for any $x \in P$ there is a $q^{l}$ that is comparable with $x$.

\section{REFERENCES}

1. J. Baumgartner and A. Hajnal, A proof (involving Martin's axiom) of a partition relation, Fund. Math. 78 (1973), 193-203.

2. P. Erdös, A. Hajnal and E. C. Milner, Set mappings and polarized partition relations, Combinatorial Theory and Its Applications. I (Proc. Colloq., Balatonfüred, 1969), Colloq. Math. Soc. János Bolyai, Vol. 4, North-Holland, Amsterdam, 1970, pp. 327-363.

3. F. Galvin, On a partition theorem of Baumgartner and Hajnal, Infinite and Finite Sets. II (Proc. Colloq., Keszthely, 1973), Colloq. Math. Soc. János Bolyai, Vol. 10, North-Holland, Amsterdam, 1975, pp. 711-729.

4. T. Jech, Set theory, Academic Press, New York, San Francisco and London, 1978.

School of Mathematics, The Institute for Advanced Study, Princeton, New Jersey 08540

Department of Mathematics, Brookiyn College (CUNY), Brookiyn, New York 11210 (Current address) 